\title{
The use of laryngeal mask airway in pediatric patient with massive post-tonsillectomy hemorrhage
}

\author{
Won Hyuk Go, Kyung-Tae Kim, Ji Yeon Kim, Won Joo Choe, and Jung Won Kim \\ Department of Anesthesiology and Pain Medicine, Ilsan Paik Hospital, Inje University School of Medicine, Goyang, Korea
}

The Laryngeal Mask Airway (LMA) is not only used in patients who have a difficult airway with a conventional laryngoscope, but it is also used in children who have a tonsillectomy to manage the airway [1]. However, cases related to the airway management of young children with massive post-tonsillectomy hemorrhage (PTH) have not been reported yet. Here, we report a case in which we have successfully performed airway management using LMA during severe ventilatory difficulty, which had occurred in a young child patient with massive PTH, in which we failed to perform an endotracheal intubation under general anesthesia.

A 6-year old female patient visited the hospital complaining of massive hematemesis. A physical examination confirmed acute oozing bleeding in the left tonsillar fossa. We decided to perform an emergent operation under general anesthesia for surgical ligature. After arrival to the operating room, we continuously carried out oral suction in the patient, who was in the trendelenburg position, in order to prevent aspiration caused by the hematemesis, while also providing her with $100 \%$ oxygen via a mask. A rapid sequence intubation (RIS) could be performed. We performed an endotracheal intubation immediately under the Sellick's maneuver, but constant intraoral bleeding inhibited us from securing a clear view, so we failed to intubate. After which, we performed a blind intubation by inserting a stylet, but we failed to do this as well. Meanwhile, the patient's oxygen saturation plunged to $79 \%$, so another doctor performed the intubation procedure, but was also unsuccessful. After which, the oxygen saturation decreased to $52 \%$, so a cricothyrotomy and tracheostomy were planned for an instant surgical airway. Because of intraoral bleeding that occurred in the tonsil and that the surgical airway was difficult due to differences in anatomical structures compared to adults, in addition, our hospital routinely prepares a LMA to general anesthesia;thus, we attempted to insert the LMA before the surgical airway. Number two of the LMA (LMA Supreme ${ }^{\mathrm{TM}}$, LMA Company Ltd, UK) was inserted immediately under the Sellick's maneuver. After the successful insertion of the LMA, ventilation was performed well without any air leakage, and the auscultation sound was normal. Oxygen saturation normalized, increasing from $48 \%$ to $100 \%$. With a Yankauer suction catheter, we suctioned by inducing pressure with a gauze on the site of bleeding, and thus, we were able to secure our view. And then, we removed the LMA, and successfully performed a RIS. A blood clot was found behind the cuff of the removed LMA (Fig. 1). After the operation, we confirmed that the patient recovered from her unconsciousness with oxygen saturation maintained at $99-100 \%$. Afterwards, since the lung sounds was normal, the patient was extubated, and was transferred to the recovery room. One hour later, the patient was then transferred to the ward since oxygen saturation was maintained at $100 \%$ without oxygenation. The results of the chest radiograph performed after the operation was normal, and the patient was discharged without any particular problems ten days later.

In general, a surgical airway is not widely used in children aged 8 years or younger in contrast to adults [2]. The reasons for this is that the cricothyroid membrane of adults is $13.7 \mathrm{~mm}$ in length and $12.4 \mathrm{~mm}$ in width on average [3], whereas the membrane of children is merely $2.6 \mathrm{~mm}$ in length and $3 \mathrm{~mm}$ in

Corresponding author: Kyung-Tae Kim, M.D., Department of Anesthesiology and Pain Medicine, Ilsan Paik Hospital, Inje University School of Medicine, Daehwa-dong, Ilsanseo-gu, Goyang 411-706, Korea. Tel: 82-31-910-7160, Fax: 82-31-910-7184, E-mail: dadalgo1@naver.com (c) This is an open-access article distributed under the terms of the Creative Commons Attribution Non-Commercial License (http:// creativecommons.org/licenses/by-nc/3.0/), which permits unrestricted non-commercial use, distribution, and reproduction in any medium, provided the original work is properly cited. 


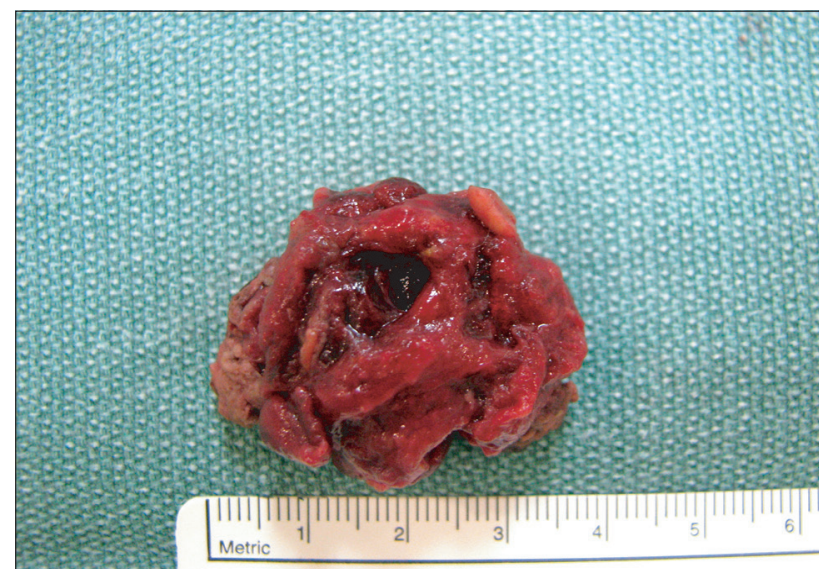

Fig. 1. Image shows that a blood clot was found behind the cuff of the removed LMA.

width [4], so unlike adults, it is a much more delicate process when performing a cricothyrotomy on children. Moreover, even if the excision succeeds, damage to the larynx can be a possibility and should not be ruled out due to their small anatomical structures [3]. In addition, the hyoid and the cricoid cartilage commonly protrude more than the thyroid cartilage in children, so a cricothyrotomy could possibly be mistakenly performed on the other site [2,4]. Besides, children have a lower capacity for residual pulmonary function and consume more oxygen per hour, so even a short time of apnea could result in hypoxia. In cases of an absence of spontaneous respiration, the risk of aspiration to the lungs, caused by acute intraoral bleeding, cannot be underestimated. For these specific cases, we prepared an apparatus for children in order to immediately perform cricothyrotomy in cases of endotracheal intubation failure;however, we attempted to insert a LMA due to the risks mentioned above and due to time constraints.

The LMA is widely used since the LMA is a ventilation method that anesthesiologists can do well during emergent situations. Moreover, since the cuff of the LMA adheres to the entry of the trachea and larynx, we thought we could prevent the bleeding that was occurring in the tonsil, outside of the cuff, from being aspirated. As a result, we successfully performed the LMA at a trial before carrying out the surgical airway, and removed the blood clot behind the cuff when we removed the LMA after securing our view with a Yankauer suction catheter (Fig. 1).

Like in this case, a LMA can be considered in which a child with massive PTH experiences an emergent situation, such as ventilatory failure or hypoxemia, that was caused due to failure of endotracheal intubation. Furthermore, the LMA should always be prepared in order to successfully manage anesthesia for patients who are not susceptible to endotracheal intubation, and for patients in which endotracheal intubation failed for the induction of general anesthesia.

\section{References}

1. Gravningsbråten R, Nicklasson B, Raeder J. Safety of laryngeal mask airway and short-stay practice in office-based adenotonsillectomy. Acta Anaesthesiol Scand 2009; 53: 218-22.

2. Coté CJ, Hartnick CJ. Pediatric transtracheal and cricothyrotomy airway devices for emergency use: which are appropriate for infants and children? Paediatr Anaesth 2009; 19 Suppl 1: 66-76.

3. Goumas P, Kokkinis K, Petrocheilos J, Naxakis S, Mochloulis G. Cricothyroidotomy and the anatomy of the cricothyroid space. An autopsy study. J Laryngol Otol 1997; 111: 354-6.

4. Navsa N, Tossel G, Boon JM. Dimensions of the neonatal cricothyroid membrane-how feasible is a surgical cricothyroidotomy? Paediatr Anaesth 2005; 15: 402-6. 